\title{
A Rupture Limit Equation for Pre-Loaded Laminated Composite Plates
}

\author{
William P. Schonberg
}

Civil, Architectural, and Environmental Engineering Department; Missouri University of Science \& Technology, 1401 North Pine Street, Rolla, MO 65409, USA; wschon@mst.edu; Tel.: +1-573-341-4787

Received: 3 January 2018; Accepted: 19 January 2018; Published: 23 January 2018

\begin{abstract}
Fiber-reinforced polymer composites offer inherent advantages over traditional metallic materials in a number of different ways; however, these materials are also highly susceptible to impact damage. In this paper, we explore the response of FRP (fiber reinforced polymer) composites under impact conditions that could result in their rupture or catastrophic failure. The work performed was aimed at developing a general, data-driven equation for initially-stressed, flat, composite plates that would differentiate between impact conditions that would result in only a hole or crack and those which would cause catastrophic plate failure or rupture. If this equation were to be subsequently shown to also model the rupture/non-rupture behavior of, for example, composite overwrapped pressure vessels, then it could also be used to appropriately tailor the design parameters and/or operating conditions of such pressurized tanks.
\end{abstract}

Keywords: high-speed impact; rupture; catastrophic failure; FRP composites

\section{Introduction}

Fiber reinforced polymer (FRP) composites offer inherent advantages over traditional materials with regard to their high strength-to-weight ratio, design flexibility, corrosion resistance, low maintenance, and extended service life. However, these materials are also highly susceptible to impact damage, which can result from any number of different low, intermediate, and high velocity impact events (e.g., bird strikes, hammer drops, sleet, orbital debris, etc.).

A wide variety of studies have been (and continue to be) performed to study the impact response of such materials. In the low to intermediate impact velocity regime, these studies typically focus on analyzing the extent of impact-induced delamination, energy partitioning, crater depth, plate fracture, etc. Reviews of the work done in this impact regime are plentiful (see, e.g., [1,2]). Alternatively, high velocity impact studies involving composite materials are much more limited. Such studies have typically examined either the use of such materials as part of a multi-wall system designed to mitigate the damage that could result from an-orbit impact by a micro-meteoroid or a piece of orbital debris, or as part of the honeycomb sandwich plates that comprise the main load-bearing walls of a satellite. A recent synopsis of the work done involving hypervelocity impact of FRP composites can be found in [3].

In addition to impact studies, other investigations have considered a variety of techniques aimed at strengthening FRP composite materials using carbon nanotubes (see, e.g., [4]), as well as carbon, ceramic, and mineral nanoparticles (see, e.g., [5]). Such studies have typically focused on improving the delamination and fatigue resistance of FRPs by enhancing, for example, their inter-laminar shear strength and fracture toughness. FRP composites have also been used to strengthen impact-damaged structural elements (see, e.g., [6]), while biocompatible composites have begun to play an important role in the development of advanced green materials (see, e.g., $[7,8]$ ).

In this paper, we explore the response of initially-stressed FRP composites plates or panels under impact conditions that are sufficiently energetic so as to result in their rupture or catastrophic 
failure. Specifically, the work performed was aimed at developing a general, data-driven equation for uni-axially loaded flat composite plates that would differentiate between impact conditions that would result in only a hole or crack and those which would cause catastrophic plate failure or rupture. The equation developed herein is an improvement over a previous version [9] in that the current version is comprised of unitless or non-dimensional terms, whereas the previous version was not. As a result, the equation developed herein is more amendable to incorporating additional, new impact test data and results for similar test conditions as they become available.

The equation to be developed is referred to as a Rupture Limit Equation (or RLE), as it is constructed to distinguish between regions of rupture and non-rupture. If this equation were to be subsequently shown to also model the rupture/non-rupture behavior of, for example, actual composite overwrapped pressure vessels, then it could also be used to appropriately tailor the design parameters and/or operating conditions of such pressurized tanks. This is an important consideration because it would allow engineers to choose design parameters and/or operating conditions so that additional sizable debris is not created as a result of an in-service high speed projectile impact.

The RLE presented herein was developed by applying multi-linear regression techniques to data obtained in three recent studies involving uni-axially stressed composite material plates. This, then, is another improvement of the RLE presented herein over the previous version, which was, in effect, merely a hand-drawn or faired curve between two sets of data points. Since the equation in this paper is statistics-based, it (and the statistics associated with it) can easily be included in a risk assessment analysis, whereas the previous version could not.

\section{Data Overview}

In this particular study, we focus on three seemingly disparate investigations involving high-speed impact tests performed on laminated graphite/epoxy plates [10-12]. Their sole common thread was that they each sought, among other things, to distinguish between impact conditions that would cause catastrophic failure of the plates and those that did not. Table 1 presents a summary of the pertinent geometric parameters/material properties of the composite panels subsequently used in the development of the RLE.

Table 1. Geometric parameters and material properties of composite panels.

\begin{tabular}{ccccc}
\hline Parameter & Reference [10] & Reference [11] & Reference [12] & Units \\
\hline \# of Tests & 43 & 46 & 53 & - \\
Material & $\mathrm{IM} / 8551-7$ & $\mathrm{~T} 300 / 5208$ & $\mathrm{~T} 300 / 934$ & - \\
Tensile Strength & $897^{\mathrm{a}}$ & 398 & 571 & $\mathrm{MPa}$ \\
Density & 1.58 & 1.60 & 1.50 & $\mathrm{~g} / \mathrm{cm}^{3}$ \\
Stacking Sequence & {$[0,90,90]_{\mathrm{s}}$} & {$\left[45_{2},-45_{2}, 0_{2}, 90_{2}\right]_{\mathrm{s}}$} & {$[ \pm 45,0,90]_{2 \mathrm{~s}}$} & - \\
Test Panel Thickness & 0.9 & $\sim 2.25$ & $\sim 2.06$ & $\mathrm{~mm}$ \\
\hline \multicolumn{5}{c}{${ }^{\text {a }}$ Estimated from single-ply properties using classic laminate theory. }
\end{tabular}

In the first study [10], test coupons were impacted by a $6.35 \mathrm{~mm}$ diameter tup with a drop weight of $1.2 \mathrm{~kg}$ that was part of a Dynatup 830 instrumented data acquisition system; impact energies ranged from approx. 0.3 to $3.0 \mathrm{~J}$. Considering the mass of the impacting tup, this energy range corresponded to an impact velocity range of 0.7 to $2.2 \mathrm{~m} / \mathrm{s}$. The uni-axially applied tensile pre-loads in these tests ranged from 0 to $\sim 34 \mathrm{kN}$. In the second and third studies [11,12], the test panels were impacted by $1.27 \mathrm{~cm}$ diameter aluminum spheres fired using a compressed air gun; impact velocities ranged from $\sim 15$ to $\sim 125 \mathrm{~m} / \mathrm{s}$, while the uni-axial tensile loads ranged from $\sim 14$ to $\sim 65 \mathrm{kN}$.

Figure 1a,b show sketches of the impact/tensile pre-loading device used in [10], and the firing mechanism used in $[11,12]$, respectively. 


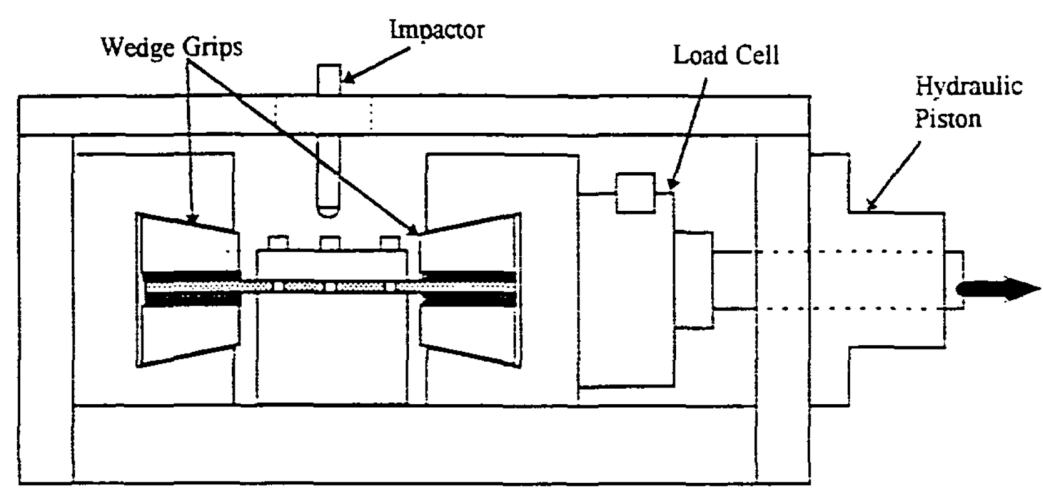

(a)

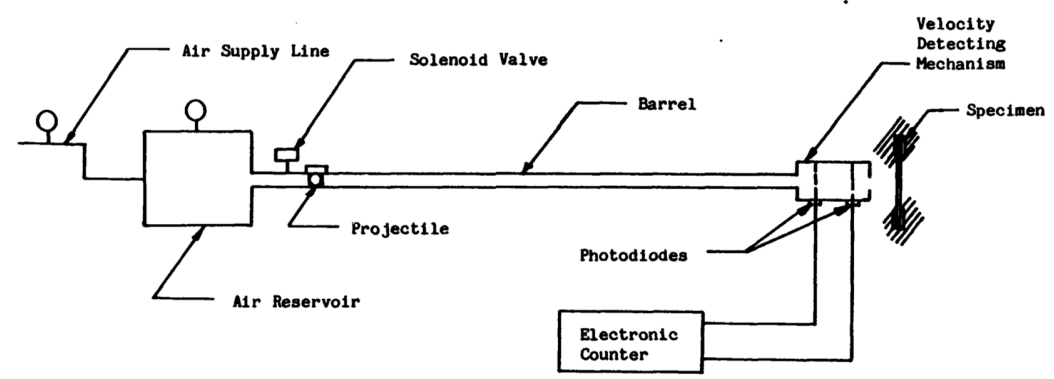

(b)

Figure 1. (a) Impact/tensile pre-loading device used in reference [10]; (b) Sketch of firing mechanism used in references $[11,12]$.

\section{Modelling the Rupture/Non-Rupture Response of Composite Plates}

The objective of the current work, then, was to ascertain whether or not the rupture/non-rupture data from the three studies described previously could be combined and then used to develop a single equation that would be capable of modelling the response characteristics evident in all three original studies. To render the equations as broadly applicable as possible, the $x$-axis was taken as the initial uni-axial stress state non-dimensionalized by the ultimate uni-axial tensile stress of the composite laminate. The impact conditions ( $y$-axis) were parameterized as impact momentum non-dimensionalized by a number of appropriate plate material properties. This approach was used successfully to model the rupture/non-rupture response of cylindrical and spherical metallic tanks [13], as well as cryogenic and room-temperature composite overwrapped pressure vessels (COPVs) under high-speed impact [14,15].

Following the successful application of this approach in these previous studies, a simple power law form was chosen for the RLE to be developed. Specifically, the power law for the curve that was intended to separate regions of rupture and non-rupture was chosen as follows:

$$
\text { Non-dimensional Momentum }=A\left(\frac{\sigma_{a p p l}}{\sigma_{\text {tenstr }}^{\text {comp }}}\right)^{B}
$$

where $\sigma_{\text {appl }}$ and $\sigma_{\text {tenstr }}^{\text {comp }}$ are the applied uni-axial stress load and the uni-axial ultimate tensile strength of the composite laminate, respectively. The non-dimensional form of impact momentum was taken to be as follows:

$$
\text { Non-dimensional Momentum }=\frac{P m_{\text {proj }} V_{\text {proj }}}{\left(\rho_{\text {comp }} t_{\text {comp }}^{3}\right) \sqrt{\frac{\sigma_{\text {tens } n \text { tr }}^{\text {comp }}}{\rho_{\text {comp }}}}}
$$


where $t_{\text {comp }}$ and $\rho_{\text {comp }}$ are the nominal thickness and mass density of the composite laminate, and $m_{p r o j}$ and $V_{\text {proj }}$ are the impactor mass and (normal or straight-on) striking velocity. In Equation (2), the first term in the denominator, therefore, has units of mass while the second has units of velocity, thereby rendering the right-hand-side of Equation (2) unitless, or non-dimensional (so long as, of course, there is consistency in the units of mass and velocity used in its numerator and denominator). The parameter $P$ was included in the numerator in Equation (2) to include the effects of the differences between the test apparatus used in the three studies. Combining Equations (1) and (2) yields the final form of the RLE as follows:

$$
\frac{P m_{\text {proj }} V_{\text {proj }}}{\left(\rho_{\text {comp }} t_{\text {comp }}^{3}\right) \sqrt{\frac{\sigma_{\text {tenstr }}^{\text {comp }}}{\rho_{\text {comp }}}}}=A\left(\frac{\sigma_{\text {hoop }}}{\sigma_{\text {ult }}^{\text {comp }}}\right)^{B}
$$

The particular forms of the non-dimensionalized pre-load and impact momentum seen in Equations (1)-(3) were motivated by the desire to include those terms that not only characterized the impact loads and pre-loading conditions, but at the same time included material parameters that were seen to differ among the test programs considered herein. As such, the terms used in Equations (1)-(3) are consistent with the identifying test specimen and material parameters shown in Table 1.

Additionally, the coefficient $P$ in Equation (3) was selected so as to allow, as much as possible, a natural separation between the ruptured and non-ruptured data points. This was intended to, in turn, facilitate the development of a RLE that would, again, as much as possible, lie between those two regions. The attractiveness and benefit of this approach is that if additional tests results were to become available, even using a different type of testing apparatus, the value of $P$ in Equation (3) could be adjusted to allow the incorporation of whatever new rupture/non-rupture data becomes available and the subsequent development of a new RLE. To this end, Table 2 presents the values of the coefficient $P$ used in the non-dimensionalization scheme defined in Equation (3):

Table 2. Values of $P$ in non-dimensionalization scheme.

\begin{tabular}{ccc}
\hline Exponent & \multicolumn{2}{c}{ Test Apparatus } \\
\hline \multirow{2}{*}{$P$} & Drop Weight [10] & Compressed Air Gun [11,12] \\
\cline { 2 - 3 } & 0.0167 & 1.0 \\
\hline
\end{tabular}

The constants $A$ and $B$ in Equation (3) are determined through a liner regression of the rupture/non-rupture (R/NR) data using the Levenberg-Marquardt algorithm [16]. This is a common algorithm for minimizing a function over the space of parameters of the function (i.e., the coefficients in a user-defined function). In addition to solving for the unknown coefficients, the algorithm also provides statistical information that can be used to assess the "goodness of fit" of the regression results as well as standard deviation curves for the regression model.

The actual regression exercise was performed by first creating a function $Z$ that is a linearized form of Equation (3) and written as follows:

$$
Z=W_{1} \ln (X)+W_{2} \ln (Y)+W_{3}
$$

where $Y$ is the non-dimensionalized projectile momentum and $X$ is the non-dimensionalized hoop stress, respectively. The function $Z$ would then take on values of +1 or -1 depending on whether a particular test resulted in either a rupture or a non-rupture. It is the constants of this linearized function $Z$ that are actually obtained through a linear regression of the $+1,-1$ "data". Once these 
constants are obtained, setting $Z=0$ yields the desired $R L E$, as well as the values of the original RLE coefficients $A$ and $B$ in terms of the parameters $W_{1}, W_{2}$, and $W_{3}$ as follows:

$$
\begin{gathered}
A=\exp \left(-\frac{W_{3}}{W_{2}}\right) \\
B=-\frac{W_{1}}{W_{2}}
\end{gathered}
$$

Table 3 contains the results of this exercise, namely, the parameters $W_{1}, W_{2}$, and $W_{3}$ (as well as their standard deviations), the coefficients $A$ and $B$, and the correlation coefficient $R^{2}$.

Table 3. Results of regression exercise.

\begin{tabular}{ccc}
\hline Parameter & Value & Standard Deviation \\
\hline $\boldsymbol{W}_{\mathbf{1}}$ & -1.4482 & 0.2367 \\
$\boldsymbol{W}_{\mathbf{2}}$ & -0.7092 & 0.2116 \\
$\boldsymbol{W}_{\mathbf{3}}$ & 0.8993 & 0.5335 \\
$A$ & 3.5543 & - \\
$B$ & -2.0421 & - \\
$R^{2}$ & $53.5 \%$ & - \\
\hline
\end{tabular}

This completes the development of the RLE for the test data considered in this study. In the next section we compare the predictions of the RLE against the test data, and offer comments on the utility and limitations of this RLE.

\section{Comparison against Empirical Results}

Figure 2 shows plots of the RLE developed herein compared to experimental rupture/no-rupture results. This figure also shows plots of \pm one standard deviation curves and \pm two standard deviation curves about the RLE curve. The red data points represent those tests which resulted in tank rupture, while the blue data points show those which did not. As can be seen in Figure 2, the regression-based RLE curve does a fairly reasonable job of modelling the rupture/non-rupture behavior of all three datasets simultaneously. There is a bit of scatter, though, as might be expected considering the difference between the methods used to generate the impact events in these three studies. This would also explain the relatively low correlation coefficient seen in Table 3.

Another means of assessing the ability of the RLE to discriminate between regions of impact parameters and operating conditions that would result in rupture from those that would not is through the use of specificity and sensitivity ratios. In the medical world, these values are used to distinguish between false positives and false negatives. For example, if we designate a rupture event as the event we are testing for, then a rupture might be considered as a "positive reading" and a non-rupture might be considered as a "negative reading". As such, the following definitions could be applied for the demarcation line associated with the RLE developed herein:

$$
\begin{gathered}
\text { Sensitivity ratio }=(\text { Actual ruptures predicted as ruptures }) /(\text { Actual ruptures } \\
\text { predicted as ruptures }+ \text { Actual ruptures predicted as non-ruptures })
\end{gathered}
$$

Specificity ratio $=($ Actual non-ruptures predicted as non-ruptures $) /($ Actual nonruptures predicted as non-ruptures + Actual non-ruptures predicted as ruptures) 


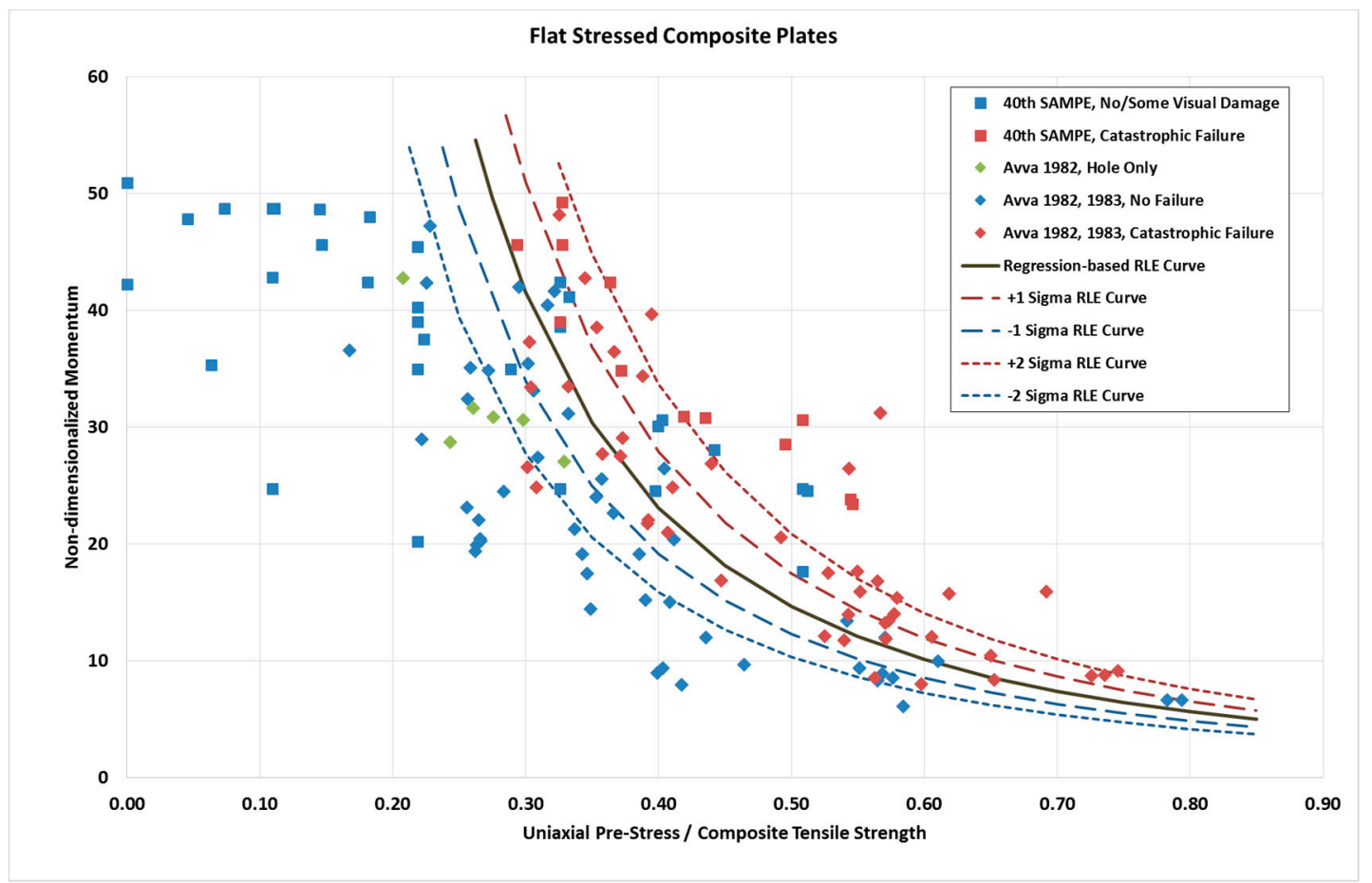

Figure 2. Plots of RLE curve and experimental rupture/no-rupture data.

If we find that we have a low specificity value (i.e., many non-ruptures predicted as ruptures) and a high sensitivity value (i.e., fewer ruptures predicted as non-ruptures), then we arguably have a fairly conservative RLE. Alternatively, if we find that we have a high specificity value (i.e., fewer non-ruptures predicted as ruptures) and a low sensitivity value (i.e., many ruptures predicted as non-ruptures), then we likely have a non-conservative RLE. Finally, if both values were found to be high, the RLE could be considered as fairly accurate, whereas if both values were low, that would indicate either a problem with the testing method or with test repeatability.

For the data considered in this study and the RLE developed based on that data, we find that we have a sensitivity value of 0.737 and a specificity value of 0.788 . Based on these results, since both values are relatively high, we can again conclude that the RLE performs fairly well in separating test results where a rupture occurred from those that resulted in non-rupture.

\section{Conclusions}

An empirical equation was developed that is able to differentiate between impact conditions that would result in only non-catastrophic damage in a stressed composite plate and those that would cause catastrophic plate failure. The equation developed was found to predict fairly well the rupture/non-rupture response of the composite plates considered. Based on the work performed, it would appear that a next step could be to determine how well the equation developed for these flat composite plates would predict the rupture/non-rupture behavior of pressurized COPVs made of similar materials. If there is a good agreement found, or if a simple correlation between the two can be found, then it might be possible to develop additional similar predictor equations without the added hazard of having to conduct very many impact tests of highly pressurized tanks.

Acknowledgments: The author wishes to extend his gratitude to the NASA Safety Engineering Center (NESC) for providing the support that made this study possible. The author also wishes to acknowledge the guidance and helpful suggestions of William E. Vesely (NASA HQ) during the course of the regression exercises in this investigation.

Conflicts of Interest: The author declares no conflict of interest. 


\section{References}

1. Greszczuk, L.B. Foreign Object Impact Damage to Composites; ASTM STP 568: Philadelphia, PA, USA, 1975.

2. Reid, S.R.; Zhou, G. Impact Behaviour of Fibre-Reinforced Composite Materials and Structures; CRC Press; Woodhead Publishing, Ltd.: Cambridge, England, 2000.

3. Schonberg, W.P. Protecting earth-orbiting spacecraft against micro-meteoroid/orbital debris impact damage using composite structural systems and materials: An overview. Adv. Space Res. 2010, 45, 709-720. [CrossRef]

4. Dikshit, V.; Bhudolia, S.K.; Joshi, S.C. Multiscale polymer composites: A review of the interlaminar fracture toughness improvement. Fibers 2017, 5, 38. [CrossRef]

5. DeCicco, D.; Asaee, Z.; Taheri, F. Use of nanoparticles for enhancing the interlaminar properties of fiber-reinforced composites and adhesively bonded joints-A review. Nanomaterials 2017, 7, 360. [CrossRef] [PubMed]

6. Tao, X.; Cao, S.; Zhang, L. Elastic stability of perforated plates strengthened with FRP under uniaxial compression. Appl. Sci. 2017, 7, 1188.

7. Bertolino, V.; Cavallaro, G.; Lazzara, G.; Merli, M.; Milioto, S.; Parisi, F.; Sciascia, L. Effect of the biopolymer charge and the nanoclay morphology on nanocomposite materials. Ind. Eng. Chem. Res. 2016, 55, 7373-7380. [CrossRef]

8. Makaremi, M.; Pasbakhsh, P.; Cavallaro, G.; Lazzara, G.; Kit Aw, Y.; Mae Lee, S.; Milioto, S. Effect of morphology and size of halloysite nanotubes on functional pectin bionanocomposites for food packaging applications. ACS Appl. Mater. Interf. 2017, 9, 17476-17488. [CrossRef] [PubMed]

9. Schonberg, W.P. Predicting the catastrophic failure of composite plates following a hypervelocity impact. In Proceedings of the 30th International Symposium on Ballistics, Long Beach, CA, USA, 11-15 September 2017.

10. Nettles, A.; Daniel, V.; Branscomb, C. The effects of tensile preloads on the impact response of carbon/epoxy laminates. In Proceedings of the 49th International SAMPE Technical Conference, Anaheim, CA, USA, 8-11 May 1995.

11. Avva, V.S. Impact Initiated Damage in Laminated Composites; AFOSR-TR-82-1038; Bolling AFB: Washington, DC, USA, 1982.

12. Avva, V.S. Fatigue/Impact Studies in Laminated Composites; AFWAL-TR-83-3060; Wright-Patterson AFB: Dayton, OH, USA, 1983.

13. Schonberg, W.P.; Ratliff, M. Hypervelocity impact of a pressurized vessel: Comparison of ballistic limit equation predictions with test data and rupture limit equation development. Acta Astronaut. 2015, 115, 400-406. [CrossRef]

14. Schonberg, W.P. Rupture of a cryogenic composite overwrapped pressure vessel following a high-speed particle impact. Aerospace 2018, in press.

15. Schonberg, W.P. Rupture of composite pressure vessels (COPVs) following a hypervelocity MMOD particle impact. In Proceedings of the 2018 AIAA SciTech Forum, Kissimmee, FL, USA, 2018.

16. PSIPlot: Scientific Spreadsheet and Technical Plotting User's Guide; Poly Software International: Pearl River, NY, USA, 2010.

(c) 2018 by the author. Licensee MDPI, Basel, Switzerland. This article is an open access article distributed under the terms and conditions of the Creative Commons Attribution (CC BY) license (http://creativecommons.org/licenses/by/4.0/). 